\title{
Large study strengthens link between autism, preterm birth
}

\author{
BY CHARLES Q. CHOI
}

11 AUGUST 2021

\section{Listen to this story:}

https://www.spectrumnews.org/wp-

content/uploads/2021/08/audio-60e3a609-9d5a-4092-b2dd-e1615d0de07d-encodings.mp3

Premature birth is tied to an increased chance of having autism, according to the largest study yet to examine the connection. And premature birth itself - rather than unrecognized genetic or environmental factors - seems to underlie the association.

The findings suggest that infants born prematurely need early evaluation and long-term follow-up to support the timely detection and treatment of autism, experts say.

"We know that early intervention can make a huge difference in later outcome, and more effective interventions are increasingly available," says April Benasich, professor of developmental cognitive neuroscience at Rutgers University in Newark, New Jersey, who did not participate in this study.

Previous research suggests that babies born preterm - before the $37^{\text {th }}$ week of pregnancy - have a roughly 30 percent higher chance of having autism than do those born full-term. Nearly 11 percent of births worldwide are premature, and more than 95 percent of these babies survive with modern neonatal care.

"Our prior work has shown that most children who were born preterm survive into adulthood without neurodevelopmental disorders or other chronic health problems," says lead investigator Casey Crump, professor and vice chair of research in the family medicine and community health department at the Icahn School of Medicine at Mount Sinai in New York City.

But researchers have long debated whether preterm birth contributes to autism or whether both 


\section{Spectrum | Autism Research News}

https://www.spectrumnews.org

conditions might share genetic or environmental influences. It was also unclear if the link shows a sex bias, or if it extends to early-term birth — during weeks 37 and 38 of pregnancy — which is roughly three times more common than preterm birth.

In the new study, researchers scoured national healthcare and birth registries to analyze data for more than 4 million people born in Sweden between 1973 and 2013.

"I was bowled over by the cohort size," Benasich says.

Both preterm and early-term birth were significantly linked to an increased likelihood of autism in both boys and girls. The earlier a baby was born, the higher her chances of having autism, the team found: About 6 percent of those born during weeks 22 to 27 of gestation have autism, compared with 2.6 percent of those born during weeks 28 to 33, 1.9 percent of those born during weeks 34 to 36, and 1.6 percent of those born during weeks 37 to 38 . By contrast, 1.4 percent of babies born full-term - 39 to 41 weeks - have autism.

The scientists detailed their findings online today in Pediatrics.

\section{Following up:}

Comparisons with siblings further revealed that the link between prematurity and autism is not primarily explained by shared genetic or environmental factors within families. Instead, prematurity itself may slightly increase an infant's chances of having autism.

"Although the relative risks of autism were significantly higher in persons born preterm than those born full-term, the absolute risk was still low - for example, only 2.1 percent of those born preterm were diagnosed with autism," Crump adds.

Prematurity could up the chances of autism via inflammation of the brain and nervous system, Crump and his colleagues suggest. Premature infants often show altered connectivity between different parts of the brain, which may also underlie the neurodevelopmental problems that are common in this group, Benasich says. Understanding how such mechanisms contribute to autism in children born preterm or early-term could shed light on the causes of autism in general, she says.

"A better understanding of the mechanisms may potentially reveal new targets for intervention at critical windows of neurodevelopment," Crump says.

Future research should collect individualized prenatal, perinatal and postnatal data to analyze what infants experience in neonatal intensive care, which could help identify key influences on autism and improve the quality of medical care, says Li-Wen Chen, clinical assistant professor of pediatrics at the National Cheng Kung University Hospital in Tainan, Taiwan, who did not take part 


\section{Spectrum | Autism Research News}

https://www.spectrumnews.org

in this work.

Though new study is limited to data from one country, Crump says, "we expect that the findings are likely to be generalizable to other settings, but they should be replicated in other populations when feasible."

Cite this article: https://doi.org/10.53053/OZCR4687 